\title{
Osculatory and Hyperosculatory Cubature Formulas
}

\author{
By Herbert E. Salzer
}

\begin{abstract}
Osculatory and hyperosculatory cubature formulas for a rectangular region, employing the function with either first, or first and second partial derivatives, were obtained together with dominant remainder terms involving higher derivatives at one point, providing exact accuracy through the fifth (seventh) degree without (with) remainders.
\end{abstract}

Osculatory and hyperosculatory cubature formulas for calculating

$$
I=\int_{y_{0}}^{y_{0}+k} \int_{x_{0}}^{x_{0}+h} f(x, y) d x d y, \quad h>0, k>0,
$$

were derived from the interpolation formulas in [1] and [2]. We have $I=F+R$, where

$$
F=(h k / D) \sum_{i, j}\left\{A_{i, j} f\left(x_{0}+i h, y_{0}+j k\right)+B_{i, j} h f_{x}\left(x_{0}+i h, y_{0}+j k\right)\right.
$$

$$
\begin{aligned}
& +C_{i, j} k f_{y}\left(x_{0}+i h, y_{0}+j k\right)+D_{i, j} h^{2} f_{x x}\left(x_{0}+i h, y_{0}+j k\right) \\
& \left.+E_{i, j} h k f_{x y}\left(x_{0}+i h, y_{0}+j k\right)+F_{i, j} k^{2} f_{y y}\left(x_{0}+i h, y_{0}+j k\right)\right\}
\end{aligned}
$$

and

$$
R=(h k / G) \sum_{r, s} G_{r, s} h^{r} k^{s} f_{x \cdots x(r \text { times }) y \cdots y(s \text { times })}\left(x_{0}, y_{0}\right)+\cdots .
$$

The osculatory formulas have no $D_{i, j}, E_{i, j}$ or $F_{i, j}$ terms in $F$. Exact integral values for the nonvanishing coefficients $A_{i, j}, B_{i, j}, \ldots$ and $D$ in (2), and the $G_{r, s}$ and $G$ corresponding to the dominant terms in (3), are tabulated in Schedules I and II. The relative accuracy in every formula $F$ increases as both $h$ and $k$ decrease. Although $R$ is essentially for estimating the error in $F$, it can also be employed to improve the accuracy in $F$ whenever higher order derivatives at $\left(x_{0}, y_{0}\right)$ are computable, even if only approximately.

All formulas and dominant remainder terms were checked exactly by numerical examples of the appropriate degree in $x$ and $y$.

Other similar formulas of generally lower order, involving considerably fewer and also lacunary interpolation conditions, but giving upper bounds to the remainders, are in [3] and [4]. In [5] the partial derivatives enter only in the single and double Laplacian at just one point.

Received September 30, 1975; revised April 26, 1976.

AMS (MOS) subject classifications (1970). Primary 65D30; Secondary 65D05.

Key words and phrases. Cubature, osculatory cubature, hyperosculatory cubature, dominant remainder terms, bivariate interpolation. 
SCHEdule I: Coefficients in Formula $F$

\begin{tabular}{|c|c|c|c|c|c|c|c|c|c|c|}
\hline $\begin{array}{r}\text { Formula } \\
\text { Coefficient }\end{array}$ & $\mathrm{O}_{2}$ & $O_{3}^{*}$ & $O_{4}^{*}$ & $O_{5}^{*}$ & $H_{2}$ & $H_{2}^{\prime}$ & $H_{2 a}$ & $H_{3}$ & $H_{3 a}^{*}$ & $H_{4}$ \\
\hline$A_{0,0}$ & 2 & 12 & 24 & 143 & 6 & 60 & & & 192 & 180 \\
\hline$A_{1,0}$ & & & 12 & 56 & & & 60 & 180 & -3888 & 180 \\
\hline$A_{0,1}$ & & & & 56. & & & & 180 & & 180 \\
\hline$A_{1,1}$ & 4 & 8 & 12 & 200 & 18 & 60 & & & & 180 \\
\hline$A_{2,0}$ & & 4 & & & & & & & & \\
\hline$A_{2,1}$ & & & 24 & & & & & & 4056 & \\
\hline$A_{2,2}$ & & & & 25 & & & & & & \\
\hline$B_{0,0}$ & & 2 & 3 & 21 & & 12 & -18 & 36 & -72 & 36 \\
\hline$B_{1,0}$ & & & -1 & -4 & & & -12 & -36 & -2322 & -36 \\
\hline$B_{0,1}$ & & & & 4 & & & & & & 36 \\
\hline$B_{1,1}$ & -1 & -4 & -29 & -80 & -6 & -12 & & & & -36 \\
\hline$B_{2,0}$ & & -2 & & & & & & & & \\
\hline$B_{2,1}$ & & & -9 & & & & & & -1650 & \\
\hline$B_{2,2}$ & & & & -7 & & & & & & \\
\hline$C_{0,0}$ & & 3 & 5 & 21 & & 12 & 30 & -24 & & 36 \\
\hline$C_{1,0}$ & & & 1 & 4 & & & & 60 & -1902 & 36 \\
\hline$C_{0,1}$ & & & & -4 & & & & -36 & & -36 \\
\hline$C_{1,1}$ & -1 & & -7 & -80 & -6 & -12 & & & & -36 \\
\hline$C_{2,0}$ & & 1 & & & & & & & & \\
\hline$C_{2,1}$ & & & 1 & & & & & & -1974 & \\
\hline$C_{2,2}$ & & & & -7 & & & & & & \\
\hline$D_{0,0}$ & & & & & & 1 & -9 & -2 & -12 & 3 \\
\hline$D_{1,0}$ & & & & & & & 1 & 3 & -664 & 3 \\
\hline$D_{0,1}$ & & & & & & & & 5 & & 3 \\
\hline$D_{1,1}$ & & & & & 1 & 1 & & & & 3 \\
\hline$D_{2,1}$ & & & & & & & & & 190 & \\
\hline$E_{0,0}$ & & & & & -1 & -3 & 10 & 20 & 56 & 5 \\
\hline$E_{1,0}$ & & & & & & & 5 & -5 & -1083 & -5 \\
\hline$E_{0,1}$ & & & & & & & & 15 & & -5 \\
\hline$E_{1,1}$ & & & & & 1 & -3 & & & & 5 \\
\hline$E_{2,1}$ & & & & & & & & & 505 & \\
\hline$F_{0,0}$ & & & & & & 1 & 5 & -12 & 90 & 3 \\
\hline$F_{1,0}$ & & & & & & & 5 & 15 & -410 & 3 \\
\hline$F_{0,1}$ & & & & & & & & 3 & & 3 \\
\hline$F_{1,1}$ & & & & & 1 & 1 & & & & 3 \\
\hline$F_{2,1}$ & & & & & & & & & 326 & \\
\hline$D$ & 6 & 24 & 72 & 480 & 24 & 120 & 60 & 360 & 360 & 720 \\
\hline
\end{tabular}


Schedule II: Coefficients in Dominant Terms of Remainder $R$

\begin{tabular}{|c|c|c|c|c|c|c|c|c|c|c|}
\hline $\begin{array}{r}\text { Formula } \\
\text { Coefficient } \\
\end{array}$ & $\mathrm{O}_{2}$ & $O_{3}^{*}$ & $O_{4}^{*}$ & $O_{5}^{*}$ & $H_{2}$ & $H_{2}^{\prime}$ & $H_{2 a}$ & $H_{3}$ & $H_{3 a}^{*}$ & $H_{4}$ \\
\hline$G_{1,1}$ & -6 & & & & & & & & & \\
\hline$G_{3,0}$ & 1 & 10 & & & & & -30 & & & \\
\hline$G_{0,3}$ & 1 & -10 & & & & & 30 & & & \\
\hline$G_{4,0}$ & & 16 & 36 & & -3 & & -6 & & & \\
\hline$G_{3,1}$ & & -5 & -20 & & & 6 & -15 & & & \\
\hline$G_{2,2}$ & & 20 & & -20 & 10 & 14 & -10 & & & \\
\hline$G_{1,3}$ & & -5 & 20 & & & 6 & 15 & & & \\
\hline$G_{0,4}$ & & -4 & 6 & & -3 & & 6 & & & \\
\hline$G_{5,0}$ & & & 45 & 1 & -2 & & -1 & & 4522 & \\
\hline$G_{4,1}$ & & & 13 & 5 & -4 & 3 & -7 & -140 & 16730 & \\
\hline$G_{3,2}$ & & & -25 & & & 10 & -5 & & 11900 & \\
\hline$G_{2,3}$ & & & 5 & & & 10 & 5 & 1400 & -11900 & \\
\hline$G_{1,4}$ & & & 13 & 5 & -4 & 3 & 3 & -840 & -16730 & \\
\hline$G_{0,5}$ & & & 3 & 1 & -2 & & 1 & & -4522 & \\
\hline$G_{6,0}$ & & & & & & & & -3 & 4442 & -12 \\
\hline$G_{5,1}$ & & & & & & & & -35 & 13461 & \\
\hline$G_{4,2}$ & & & & & & & & -105 & 770 & -140 \\
\hline$G_{3,3}$ & & & & & & & & 525 & -31675 & \\
\hline$G_{2,4}$ & & & & & & & & 245 & -37170 & -140 \\
\hline$G_{1,5}$ & & & & & & & & -315 & -15799 & \\
\hline$G_{0,6}$ & & & & & & & & -3 & -2264 & -12 \\
\hline$G_{7,0}$ & & & & & & & & & & -6 \\
\hline$G_{6,1}$ & & & & & & & & & & -6 \\
\hline$G_{5,2}$ & & & & & & & & & & -70 \\
\hline$G_{4,3}$ & & & & & & & & & & -70 \\
\hline$G_{3,4}$ & & & & & & & & & & -70 \\
\hline$G_{2,5}$ & & & & & & & & & & -70 \\
\hline$G_{1,6}$ & & & & & & & & & & -6 \\
\hline$G_{0,7}$ & & & & & & & & & & -6 \\
\hline$G$ & 72 & 720 & 4320 & 14400 & 1440 & 720 & 720 & 30240 & 00302400 & 1209600 \\
\hline
\end{tabular}

In Schedules I and II, the letter $O$ or $H$ denotes osculatory or hyperosculatory, and the subscript gives the number of nodes $x_{0}+i h, y_{0}+j k$. The letter $a$ denotes an alternative configuration of nodes, while the prime $\left({ }^{\prime}\right)$ denotes a different formula for the same nodal configuration. The asterisk (*) notes a formula having a node external to the rectangle $\left(x_{0}, y_{0}\right),\left(x_{0}+h, y_{0}\right),\left(x_{0}+h, y_{0}+k\right),\left(x_{0}, y_{0}+k\right)$.

Comments. (1) Including $H_{3 a}^{*}$, when $H_{3}$ has much smaller coefficients in both $F$ and $R$, is justifiable because it illustrates the big difference made by one irregularly located external node, and also, because it could serve as a checking formula for $\mathrm{H}_{3}$. 
(2) The great advantage in $O_{5}^{*}$ over $O_{4}^{*}$, even though the former uses more data, is apparent from Schedule II in which there are ten remainder terms for $O_{4}^{*}$ and only five much smaller remainder terms for $O_{5}^{*}$.

(3) $H_{4}$ is the most appealing formula, having the highest accuracy and stability, combining small coefficients with complete symmetry.

In considering the compounding of formulas (2) and (3) for cubature over more than one rectangle, it should be realized that they are based upon interpolation formulas that were designed to produce minimal dominant remainder terms for $(x, y)$ not too far from $\left(x_{0}, y_{0}\right)$. To achieve this it was necessary in some cases to have one node outside the rectangle. Also, since the dominant remainder (3) is in terms of higher derivatives at a single point, the remainder after compounding over more than four rectangles requires at least some estimates for the higher derivatives at points other than $\left(x_{0}, y_{0}\right)$. That is the case if the compounding is applied to form a single strip of three or more rectangles in either the $x$ - or $y$-direction. Such single-strip formulas, when they involve no points outside the width of the strip of rectangles, where the nodes would have simple repeating configurations, would be analogous to a multiinterval Simpson-type of formula; and they can be derived from every $\mathrm{O}$ - and $\mathrm{H}$ formula except $\mathrm{O}_{5}^{*}$. The nodes for compound $\mathrm{H}_{2}$ or $\mathrm{H}_{2}$, would be on just one line. All single-strip formulas may be combined widthwise.

The most convenient compound formulas are those formed for two to four rectangles suitably oriented, because the dominant remainder terms will still involve the higher derivatives at the single point $\left(x_{0}, y_{0}\right)$. This is particularly applicable to the $H$ formulas. We may compound $H_{2}$ or 2', $H_{2 a}, H_{3}$ and $H_{4}$ over four rectangles centered at $\left(x_{0}, y_{0}\right)$ for respectively 5-, 3-, 5- and 9-point formulas. In the compounding, $h$ and $k$ must be kept positive in going from quadrant to quadrant, while the signs of the derivatives change or the variables $x$ and $y$ are interchanged.

For four-rectangle compound formulas the new $A$ to $F$ coefficients are obtained by mixed additions of those in Schedule $I$ which, in the suitably oriented first to fourth quadrants, are: $A, A, A, A ; B,-C,-B, C ; C, B,-C,-B ; D, F, D, F ; E,-E, E,-E ; F, D, F$, $D$. From Schedule II we obtain the new $G_{r, s}$ 's which are, for $r, s$ both even, $G_{r, s}+$ $G_{s, r}+G_{r, s}+G_{s, r}=2\left(G_{r, s}+G_{s, r}\right)$; for $r$ even, $s$ odd, $G_{r, s}+G_{s, r}-G_{r, s}-G_{s, r}=0$; for $r$ odd, $s$ even, $G_{r, s}-G_{s, r}-G_{r, s}+G_{s, r}=0$; for $r$, s both odd, $G_{r, s}-G_{s, r}+G_{r, s}$ $-G_{s, r}=2\left(G_{r, s}-G_{s, r}\right)$.

To illustrate on $\mathrm{H}_{3}$, from the preceding relations we obtain for the nonvanishing new $A$ to $F$ coefficients, for $(i, j)$ in the order $(0,1),(1,0),(0,-1),(-1,0): 360,360$, 360,$360 ;-60,-72,60,72 ;-72,60,72,-60 ; 20,6,20,6 ; 20,-20,20,-20 ; 6,20,6,20$; also $D_{0,0}=F_{0,0}=-28$. The nonvanishing new $G_{r, s}$ 's are $G_{6,0}=G_{0,6}=-12$ and $G_{4,2}$ $=G_{2,4}=280$. The denominators $D$ and $G$ are unchanged.

1. H. E. SALZER \& G. M. KIMBRO, Tables for Bivariate Osculatory Interpolation Over a Cartesian Grid, Convair Division of General Dynamics, San Diego, Calif., 1958, 40 pp. MR 20 \#5550. 
2. H. E. SALZER, "Formulas for bivariate hyperosculatory interpolation," Math. Comp., v. 25, 1971, pp. 119-133. MR $44 \# 4874$.

3. D. V. IONESCU, "Generalization of the quadrature formula of $N$. Obreschkoff for the double integral," Acad. R. P. Romîne Fil. Cluj. Stud. Cerc. Mat., v. 13, 1962, pp. 35-86. MR 27 \#4360.

4. D. V. IONESCU, "Generalization of the quadrature formula of N. Obreschkoff for double integrals," Stud. Cerc. Mat., v. 17, 1965, pp. 831-841. MR 34 \#5285.

5. S. E. MIKELADZE, “On formulas for mechanical cubatures, containing partial derivatives of the integrand," Bull. Acad. Sci. Georgian SSR, v. 4, 1943, pp. 297-300. (Russian) MR 6, 133. 